\title{
THE USE OF RAMAN SPECTROSCOPY TO MONITOR THE REMOVAL OF HUMIC SUBSTANCES FROM CHARCOAL: QUALITY CONTROL FOR ${ }^{14} \mathrm{C}$ DATING OF CHARCOAL
}

\author{
Dani Alon ${ }^{1} \cdot$ Genia Mintz $^{2} \bullet$ Illit Cohen ${ }^{1} \bullet$ Steve Weiner $^{1} \bullet$ Elisabetta Boaretto $^{2,3}$
}

ABSTRACT. One of the largest sources of uncertainty in radiocarbon dating stems from the sample pretreatment procedures used to minimize contamination. A major source of carbon contamination in charcoal from archaeological sites is humic substances carried by groundwater. Here we present a method, independent of ${ }^{14} \mathrm{C}$ dating itself, to evaluate the effectiveness of the cleaning procedure of charcoal. Raman spectra of mixtures of humic substances (HS) and laboratory prepared charcoal indicate that Raman spectroscopy can be used as a semi-quantitative measure of the amount of humic substances associated with archaeological charcoal. Raman spectral analysis of archaeological charcoal samples subjected to different cleaning regimes supports this contention. Such measurements can provide quality control for charcoal preparation procedures and may assist in the interpretation of carbon-dating results.

\section{INTRODUCTION}

Charcoal is one of the most important materials used for dating in archaeology. Charcoal from archaeological sites ("archaeological charcoal") is composed of the charred organic material itself and its diagenetic decomposition products. It may also contain associated humic substances (HS) thought to be derived from groundwater, and adhering carbon-containing sediments. For the purposes of radiocarbon dating, the latter two components are considered contaminants of the charcoal, as they may contain relatively large amounts of carbon that do not necessarily originate from the same time as the plant material from which the charcoal was derived. The chemical structure of the charcoal itself varies considerably according to the type of burnt material, the conditions of combustion, and the preservational environment following deposition and burial (Darmstadt et al. 2000; Guo and Bustin 1998; Nishimiya et al. 1988). Archaeological charcoal is thus a heterogeneous material, and the removal of contaminating carbon-containing components is a challenging problem.

The approach used by most ${ }^{14} \mathrm{C}$ laboratories to solve this problem is to treat the samples first with hydrochloric acid to remove associated carbonates and carbonated apatite, followed by repeated treatments with a dilute solution of sodium hydroxide to remove the humic substances. Finally, the sample is washed again in acid to remove absorbed carbon dioxide, and then dried. This is referred to as the acid-alkali-acid or "AAA procedure" (Olson and Broecker 1958; Hatté et al. 2001). The specific conditions used vary between laboratories and between samples, and the effectiveness of the treatment is usually subjectively evaluated by the absence of color in the extracting alkali solvent solution. Carbon containing material such as clay may be removed during washing, but is not usually specifically separated

Several solutions have been proposed to address the HS contamination problem, but these are not independent of the ${ }^{14} \mathrm{C}$ measurement itself. One approach is to carbon-date the material extracted during the alkali treatment in order to evaluate whether or not it is indeed a source of contamination (Olson and Broecker 1958). Another is to combust the organic material to be dated at different temperatures, choosing the appropriate fraction (McGeehin et al. 2001; Bird et al. 1999). To date, no independent analytical method exists that can be used to monitor the effectiveness of the AAA treatment. Thus there are also no independent means of assessing the reliability of the ${ }^{14} \mathrm{C}$ age with

\footnotetext{
${ }^{1}$ Department of Structural Biology, Weizmann Institute of Science, 76100 Rehovot, Israel

${ }^{2}$ Department of Environmental Sciences and Energy Research, Radiocarbon Dating Laboratory, Weizmann Institute of Science, 76100 Rehovot, Israel

${ }^{3}$ Corresponding author. Email: elisabetta.boaretto@weizmann.ac.il.
} 
respect to the purity of the charcoal sample. This is in contrast to ${ }^{14} \mathrm{C}$ dating of fossil collagen, where two different methods for independently assessing purity exist.

The $\mathrm{C} / \mathrm{N}$ ratio of collagen differs from most other proteins and certainly from other common forms of organic materials. It is thus a useful criterion for assessing collagen purity (DeNiro 1985). The collagen infrared spectrum is also distinctive and can be used to assess collagen purity (DeNiro and Weiner 1988).

Raman spectroscopy should be ideal for independently assessing charcoal purity as graphite, a major component of charcoal, does have a distinctive Raman spectrum (Tuinstra and Koenig 1970) and HS tend to fluoresce strongly (Yang and Wang 1997). It is also nondestructive and requires very small amounts of material. Unlike most sediment from archaeological sites, we noted that charcoal samples do produce a Raman spectrum that is not overwhelmed by fluorescence. We thus propose the use of Raman spectroscopy as an independent means of "quality control" that can be used to assess the effectiveness of the AAA procedure. The method presented here provides a semi-quantitative assessment of the amount of humic substances associated with charcoal from archaeological sites.

\section{MATERIALS}

Graphite: spectroscopic grade graphite rods produced by United Carbons.

Modern charcoal: pine wood without the bark (Pinus halepensis) burnt by us in an open fire (maximum $\mathrm{T}=850{ }^{\circ} \mathrm{C}$ ) on a clean rocky substrate. This modern charcoal, when treated with $1 \%$ $\mathrm{NaOH}$, did not produce a colored extract solution, supporting our assumption that the modern charcoal is HS free, or itself does not produce an alkali extractable fraction.

Humic Acid: A large amount of HS was extracted ( 10 g) from burnt wheat grains excavated in Tel Hadar dating from the Iron Age, by treating the powdered grains for 20 minutes with $1 \% \mathrm{NaOH}$. The solution was then filtered through a 0.8 -micron Millipore filter and centrifuged at $2860 \mathrm{~g}$ for $10 \mathrm{~min}$. in order to remove all solids. $1 \mathrm{~N} \mathrm{HCl}$ was added and the $\mathrm{HS}$ was retrieved by centrifugation and washing in water as described above. The HS was dried at $60^{\circ} \mathrm{C}$, and then crushed into a fine powder using an agate mortar and pestle.

Archaeological wood charcoal samples, chosen to represent various ages and preservation environments, were obtained from sites listed in Table 1.

Table 1 Archaeological samples used

\begin{tabular}{lll}
\hline Name of site & ${ }^{14} \mathrm{C}$ dates $(\mathrm{BP})$ & Description \\
\hline Tel Lachish & $2500 \pm 25$ & Crumbly, not shiny, wood fibers not visible \\
Qumran & $8740 \pm 30$ & Shiny, visible fibers \\
Tel Hazor & $3395 \pm 25$ & Shiny, visible fibers \\
Tel Keissan & Undated & Shiny, visible fibers \\
\hline
\end{tabular}

\section{METHODS}

\section{Sample Preparation}

Archaeological charcoal can be found in many sizes varying from macroscopic pieces that maintain the original structures of the burnt material, to microscopic particles with poorly defined structure. 
They also presumably vary with respect to their degree of burning and their state of preservation. In order to obtain reproducible measurements, charcoal samples must be homogenized, thus overcoming sampling problems such as differences in chemical composition between the outer and inner parts of each piece or many small pieces that originated from different materials burnt together. Homogenization was achieved by grinding the samples to a fine powder (particle size $<25 \mu \mathrm{m}$ ), using an agate pestle and mortar.

Thorough mixing of modern charcoal and HS mixtures was achieved by preparing each substance as described above. After the powders were weighed and placed in a vial, rough mixing was attained using a vortex, followed by fine grinding and thorough mixing in an agate mortar and pestle.

All cleaning procedures are based on the following AAA technique. $50 \mathrm{mg}$ mixtures were placed into $15 \mathrm{~mL}$ polypropylene vials. The carbonates and carbonated apatite were first removed using $10 \mathrm{~mL}$ of $1 \mathrm{~N} \mathrm{HCl}$ for $20 \mathrm{~min}$ at room temperature. This process was repeated if bubbling continued. The samples were washed twice with distilled water and centrifuged at $2860 \mathrm{~g}$ for $5 \mathrm{~min}$. The pellet was resuspended in $10 \mathrm{~mL}$ of $1 \% \mathrm{NaOH}$ for $20 \mathrm{~min}$ at room temperature to extract $\mathrm{HS}$. The charcoal was separated from the HS by centrifugation (as described above) and this was repeated until the supernatant remained clear. The $\mathrm{NaOH}$ dissolves atmospheric $\mathrm{CO}_{2}$ into the solution. This carbon source was removed by repeating the hydrochloric acid treatment. The pellet was finally washed with water and placed in an oven at $60{ }^{\circ} \mathrm{C}$ until dry.

During the drying step of the procedure a pellet is formed. This pellet was crushed to a fine powder using an agate mortar and pestle. The Raman spectra of all samples, whether cleaned or not, were measured when the sample was in the state of a fine powder. The powder was placed into small depressions ( $3 \mathrm{~mm}$ diameter, $1 \mathrm{~mm}$ deep at center) drilled into an aluminum plate. To form the relatively smooth surface needed for the measurement, the powder surface was lightly pressed by hand using a small brass piston.

\section{Raman Measurements}

Measurements were made in air at room temperature using a Renishaw 2000 Raman Imaging Microscope through a $50 \times$ lens without a polarizer. The excitation at $632 \mathrm{~nm}$ was produced by a $25 \mathrm{mw} \mathrm{HeNe}$ laser. The guiding principle used while defining the settings of the Raman spectrograph was to find the best trade off between the precision of the measurement and the time required for making the analysis. In our measurements each sample was measured five to ten times in different places, being careful to avoid the sample holder edges. Each measurement consisted of three scans in the minimal range set around $1600 \mathrm{~cm}^{-1}\left(1200-2000 \mathrm{~cm}^{-1}\right)$, which we found to be the relevant part of the spectrum. The spectral resolution was $4 \mathrm{~cm}^{-1}$.

Special attention must be given to sources of interference. Two such effects were found to be most important. The first is fluorescence that is produced during sample preparation; exposure to UV light, and changing thermal conditions affect the measured values. This problem was overcome by keeping all samples under exactly the same conditions of lighting and temperature. Simultaneous preparation of all the samples is required

The second effect enters into the measurement itself. The material used as a sample holder, aluminum in our experiment, may contribute to the spectrum. This background spectrum must be measured beforehand, and prevented from having any effect by avoiding the edges of the sample holder. The measurements must also be done in complete darkness, since external light sources such as the computer monitor, may affect the spectra. 


\section{RESULTS}

The Raman effect arises when electromagnetic radiation irradiates a molecule, which subsequently scatters the light. While most of this scattered light is at the same wavelength as the incident light, some is scattered at a different wavelength. This inelastically scattered light is called Raman scatter. It results from the bonds changing their energy states (Colthup et al. 1990). Complex substances that contain multiple types of bonds will produce broad emission peaks, such is the case of charcoal and HS. Pure substances, which contain few well-defined bonds such as graphite, produce discrete peaks, which can be used as a fingerprint of the substance measured.

A comparison of the Raman shift spectra of pure synthetic graphite, modern charcoal and an extract of HS shows that all three materials share two similar peaks (around 1300 and $1600 \mathrm{~cm}^{-1}$ ) (Figure 1). The peak around $1600 \mathrm{~cm}^{-1}(\mathrm{G})$ is characteristic of graphite and appears in all graphitic materials (Yang and Wang 1997). The $1300 \mathrm{~cm}^{-1}$ peak (D) is attributed to a particle size effect. Measuring the intensity ratio $\mathrm{I}(\mathrm{D}) / \mathrm{I}(\mathrm{G})$ provides information regarding the structural properties of the material (Tuinstra and Koenig 1970; Mennella et al. 1994). The fact that none of these materials has a unique Raman peak implies that the materials have a common structural basis, and that we cannot use Raman spectroscopy to evaluate varying proportions of HS by simply comparing ratios of different peaks.
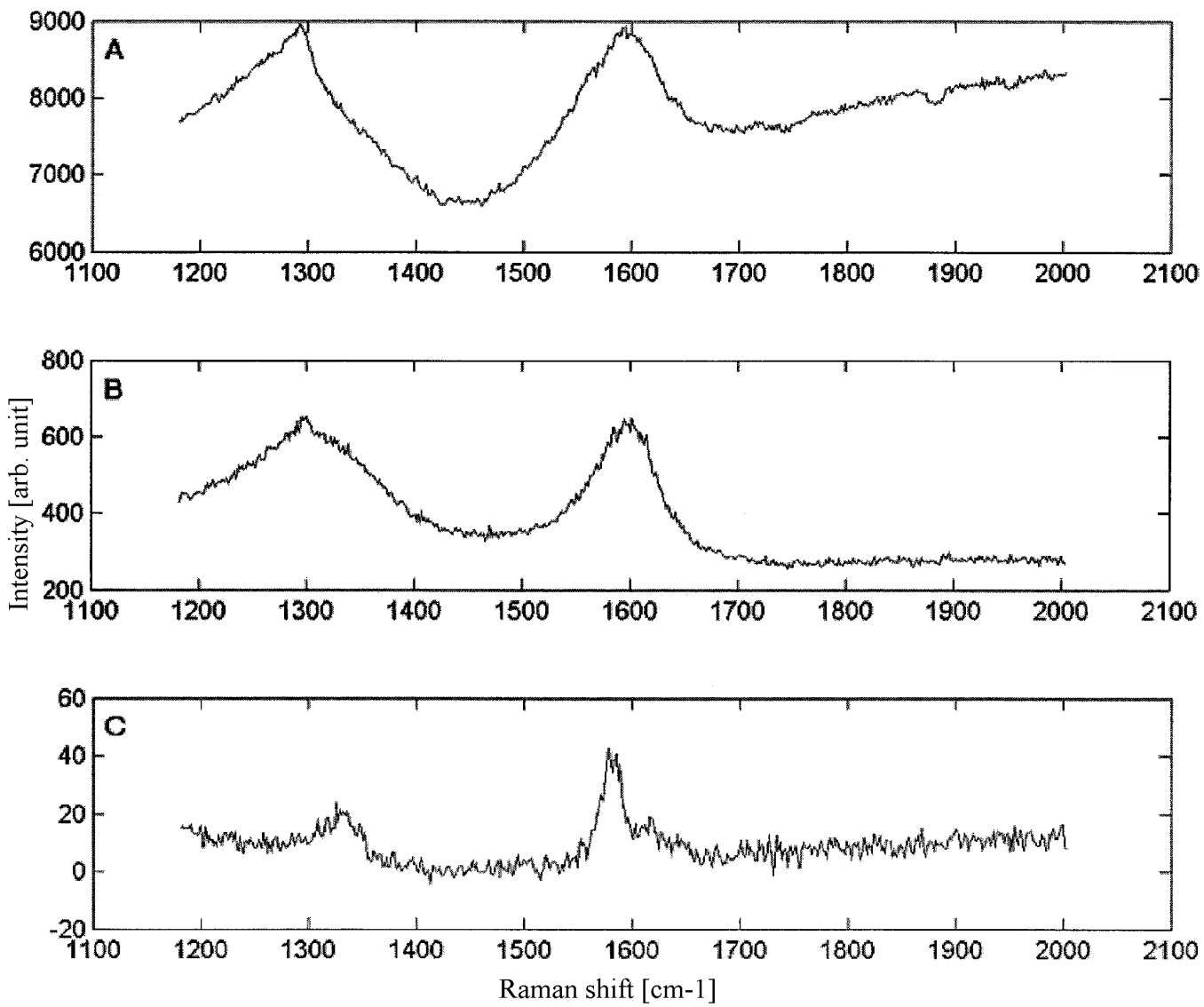

Figure 1 Raman spectra of (A) humic substance used in our experiment, (B) laboratory prepared modern charcoal, and (C) pure graphite. These three spectra share similar peaks at $\sim 1300$ and $\sim 1600 \mathrm{~cm}^{-1}$. Mean intensity and the slope of the spectra between 1700 and $1900 \mathrm{~cm}^{-1}$ are the main differentiating factors. 
The Raman shift spectra of the three materials do however differ with respect to the level of fluorescence (Figure 1). The intensity of emitted light from the humic substances is considerably greater than that of the modern charcoal and graphite. Another differentiating factor is the slope of the spectra between 1700 and $1900 \mathrm{~cm}^{-1}$. In both graphite and modern charcoal the slope in this part of the spectrum is close to zero, while the humic substances spectrum has a positive slope. The third difference is the exact position of the G peak. The peak maxima of graphite and HS differ by about $10 \mathrm{~cm}^{-1}$, with charcoal being intermediate. Other parameters, such as peak height ratios and peak shapes regarding different base lines, were also measured. In order to determine which, if any, of these parameters could be used as semi-quantitative measures for HS content, different mixtures of modern charcoal and HS were tested. Five samples were prepared by mixing different masses of powdered modern charcoal with powdered HS.

The Raman spectrum of each mixture was measured ten times producing the results shown in Figure 2. The reproducibility of individual analyses is not good, but when all the spectra are superimposed using one ordinate scale (Figure 2, lower right), it is clear that they roughly reflect the varying proportions of HS in the samples.

We therefore measured the average intensity and slope of each set of spectra. The mean intensity of each spectrum was acquired by summing the intensity values measured by the spectroscope and dividing by their number (or the area under the spectrum divided by its width). The slope was calculated using a linear fit (least squares) to the curve between 1700 and $1900 \mathrm{~cm}^{-1}$. The 10 calculated values of intensity and slope for each mixture were averaged and their standard deviations were used as errors. The results are shown in Figure 3.

Both plots show a clear linear correlation ( $R$ factor of Figure $3 A=0.991$; $R$ factor of Figure $3 \mathrm{~B}=0.989$ ) between the measured parameter and the mass percent of HS. Each parameter (mean intensity or slope) can thus be independently used as an indicator for the presence of HS. When the amount of HS is not known, such as in archaeological samples, each parameter can provide a one-dimensional scale. In this case, we recommend using the slope parameter since its value can be easily obtained using a ruler. If both parameters are measured, they can be plotted one against the other producing a two-dimensional plot independent of the mass percent of HS. The advantage of the two-dimensional measurement is that internal consistency can be better assessed, and that experimental errors are more likely to appear in one of the parameters rather than in both of them simultaneously. These errors will be expressed as a point off the normal linear fit, indicating that something is wrong or unexpected.

The changing position of the $\mathrm{G}$ peak was also calculated for the different mixtures, but the effect was found to be unreliable, to the extent that different methods to determine peak maxima (de-convolution and parabolic fit to peak summit) produced different results. The results indicated a rough correlation between the amount of HS and the position of the G peak, but the difficulty of calculating this parameter and the weakness of the effect, dissuaded us from further use of this parameter.

The next step was to determine whether the results obtained using mixtures of freshly prepared charcoal and one HS preparation are relevant to real archaeological samples subjected to the AAA treatment. We chose to do this empirically by measuring the mean intensities and slope of archaeological charcoal samples from various ages and preservation environments (Table 1) after only $\mathrm{HCl}$ treatment, and comparing them to the same samples treated after one, two or more cycles of $\mathrm{NaOH}$ treatment following the AAA procedure. A modern charcoal sample as well as four archaeological samples were homogenized and divided into three portions A, B, and C. The samples were treated as described in Table 2. 

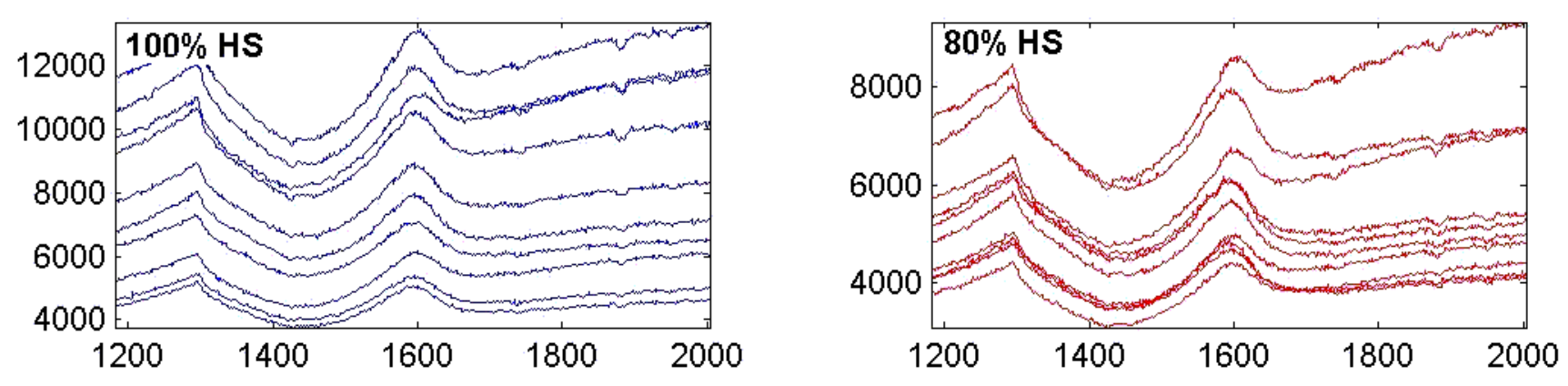

5
0
0
0
0
0
0
0
$a$
$a$
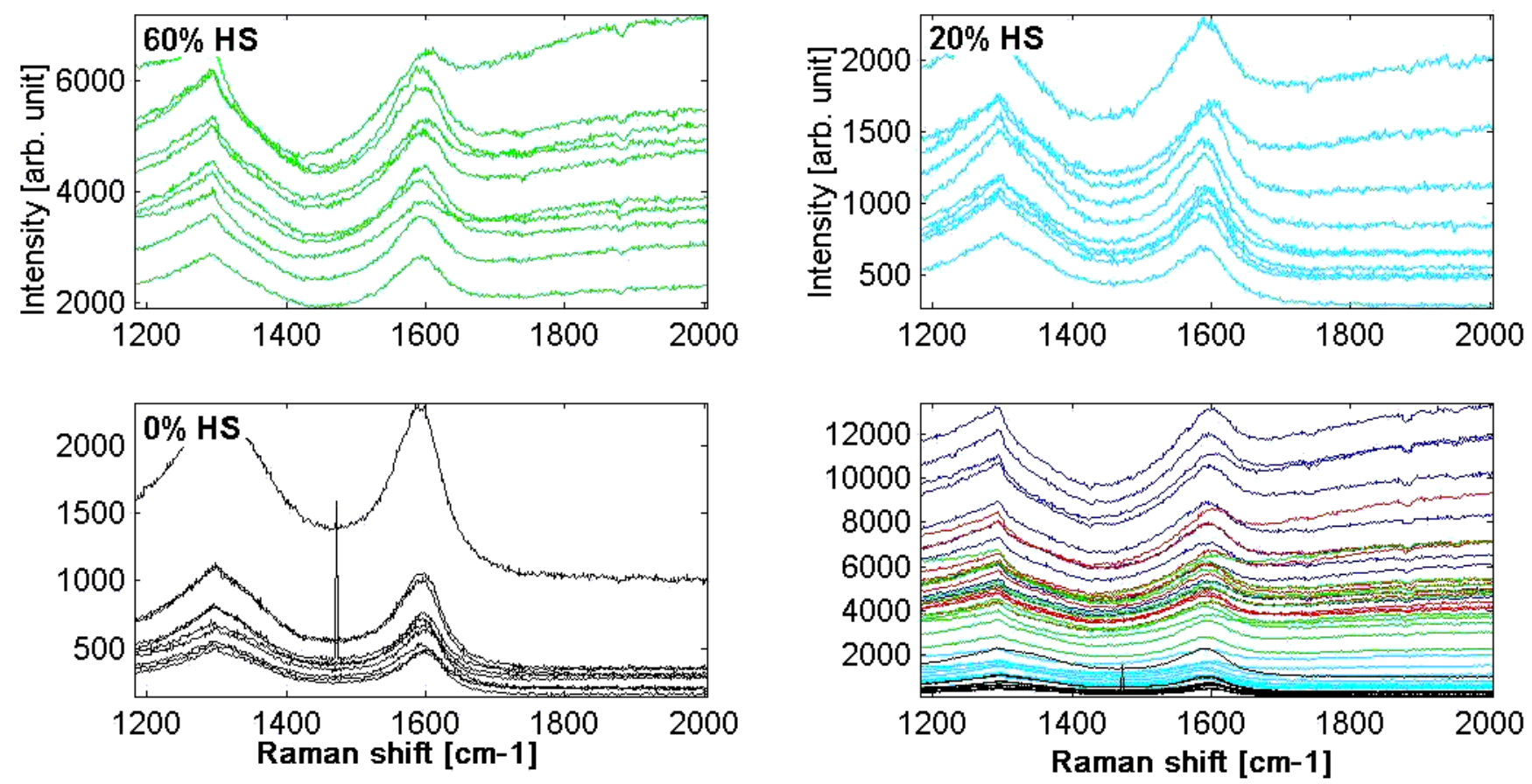

Figure 2 Raman spectra of modern charcoal and varying amounts of HS. Figure on lower right shows all the spectra superimposed on one ordinate scale. Note the correlation between the amount of HS and the intensity and slope of the Raman spectra. 

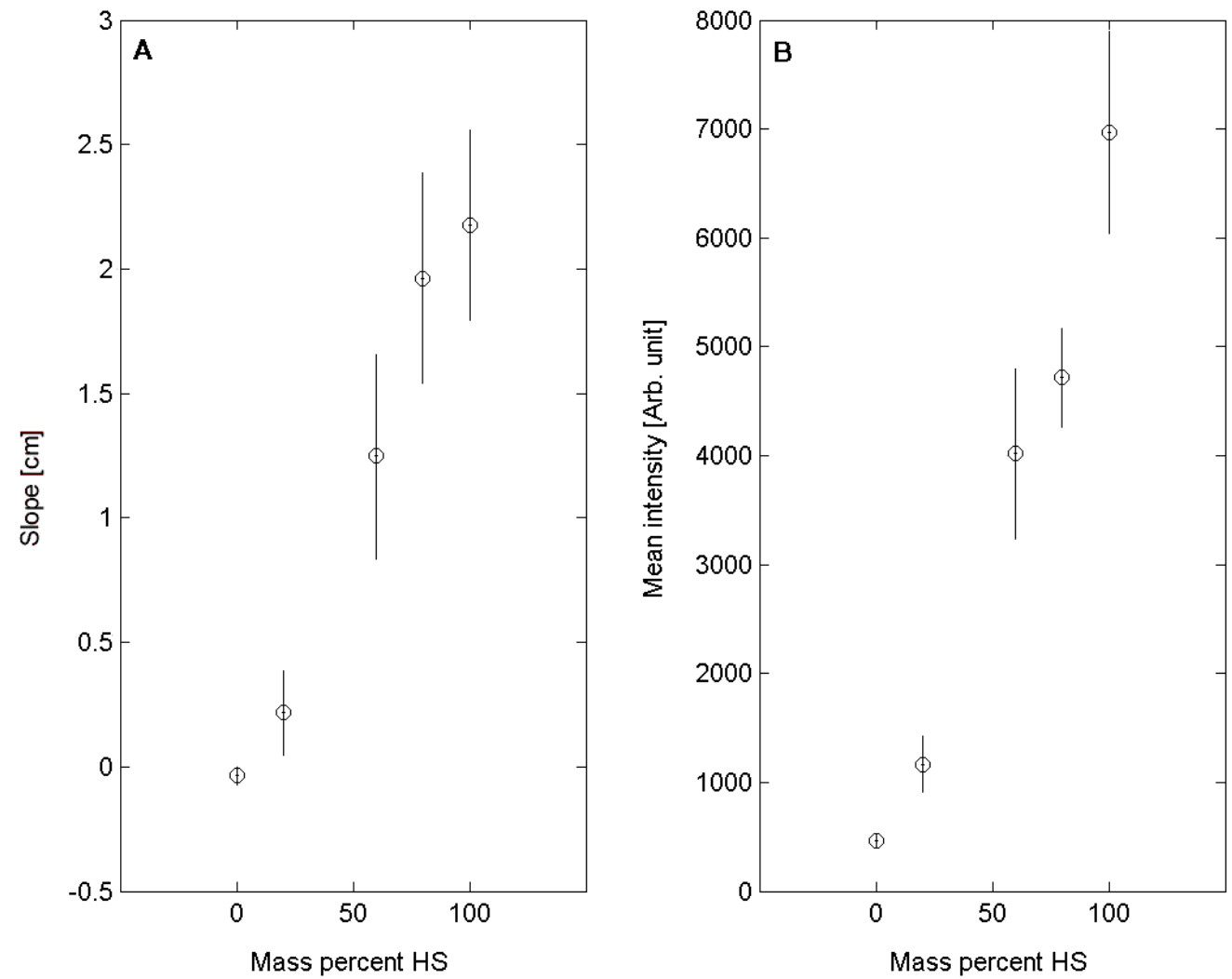

Figure 3 Plot of the slope (A) and mean intensity (B) as a function of mass percent HS. Each value is the mean of 10 measurements $(n=10 \pm \operatorname{std}$ dev $)$

The first fraction (A) of each sample was treated only with $\mathrm{HCl}$. The second (B) was cleaned once with $\mathrm{NaOH}$ followed by $\mathrm{HCl}$, while the third (C) went through the complete AAA procedure (as described in the methods) until it was judged to be clean based on the visual color test normally used. The results are shown in Figure 4. All the points fall on the same curve irrespective of the source of the sample. We note also that the mixture of modern charcoal and varying proportions of HS (magenta stars) also fall on the same curve. We thus conclude that the measurements do indeed monitor the effect of the AAA treatment on archaeological charcoal. When individual samples are tested (Figure 5) it can be seen that with each simulative cycle of the AAA procedure, the points move "down" the curve in the direction of modern charcoal. This effect can be used to empirically monitor HS removal.

\section{DISCUSSION}

Raman spectroscopy can be used to monitor the effectiveness of the AAA procedure in removing HS contamination from archaeological charcoal. The main advantage of this method is that Raman measurements of charcoal samples are made through a microscope, allowing the use of milligram amounts of material without destroying or even touching the sample. The same samples can then be used for ${ }^{14} \mathrm{C}$ analysis. The disadvantage is the reproducibility resulting in only a semi-qualitative estimate of HS contamination. 
Table 2 Sample treatment procedures used to purify modern and archaeological charcoal

\begin{tabular}{|c|c|c|c|c|c|c|c|c|c|c|c|}
\hline \multirow{2}{*}{ Sample } & \multicolumn{3}{|c|}{$\mathrm{HCl}$} & \multirow{2}{*}{$\begin{array}{c}\mathrm{NaOH} \\
1 \% \\
\end{array}$} & \multirow{2}{*}{$\begin{array}{c}\mathrm{NaOH} \\
1 \% \\
\end{array}$} & \multirow{2}{*}{$\begin{array}{c}\mathrm{NaOH} \\
1 \% \\
\end{array}$} & \multirow{2}{*}{$\begin{array}{c}\mathrm{NaOH} \\
1 \% \\
\end{array}$} & \multirow{2}{*}{$\begin{array}{c}\mathrm{NaOH} \\
1 \% \\
\end{array}$} & \multicolumn{3}{|c|}{$\mathrm{HCl}$} \\
\hline & & $1 \mathrm{~N}$ & $\mathrm{H}_{2} \mathrm{O}$ & & & & & & $\mathrm{H}_{2} \mathrm{O}$ & $1 \mathrm{~N}$ & $\mathrm{H}_{2} \mathrm{O}$ \\
\hline \multirow[t]{3}{*}{ Modern charcoal } & A & + & + & & & & & & & & \\
\hline & B & + & + & + & & & & & + & + & + \\
\hline & $\mathrm{C}$ & + & + & + & + & & & & + & + & + \\
\hline \multirow[t]{3}{*}{ Hazor } & A & + & + & & & & & & & & \\
\hline & B & + & + & + & & & & & + & + & + \\
\hline & $\mathrm{C}$ & + & + & + & + & & & & + & + & + \\
\hline \multirow[t]{3}{*}{ Keissan } & A & + & + & & & & & & & & \\
\hline & $\mathrm{B}$ & + & + & + & & & & & + & + & + \\
\hline & $\mathrm{C}$ & + & + & + & + & + & + & & + & + & + \\
\hline \multirow[t]{3}{*}{ Qumran } & A & + & + & & & & & & & & \\
\hline & B & + & + & + & & & & & + & + & + \\
\hline & $\mathrm{C}$ & + & + & + & + & + & & & + & + & + \\
\hline \multirow[t]{3}{*}{ Lachish } & A & + & + & & & & & & & & \\
\hline & $\mathrm{B}$ & + & + & + & & & & & + & + & + \\
\hline & $\mathrm{C}$ & + & + & + & + & + & + & + & + & + & + \\
\hline
\end{tabular}

Perhaps the most important practical observation is that all the measurements of archaeological charcoal samples treated to different extents by the AAA procedure fall on the same curve. Furthermore, this curve is very similar to the curve calculated from the modern charcoal and HS mixtures discussed above (Figure 4). This occurs irrespective of source, age, and the degree of contamination of all the different archaeological samples measured. Thus the method is applicable to a variety of archaeological samples.

When the progression of individual samples are monitored (Figure 5), it is clear that with additional treatment of $\mathrm{NaOH}$, the intensity and slope values decrease in a consistent manner, thus showing that the method does monitor the effectiveness of the AAA treatment. Furthermore, in some samples, e.g. Tel Keissan, the method shows that additional AAA treatment will be ineffective and that the sample, at least in this respect, is clean. In contrast the Lachish sample, whose initial Raman spectra suggested the presence of the highest values of HS, may still be contaminated.

The Tel Hazor measurement produced an unexpected result. While fractions A and B seemed normal, fraction C "jumped" back up the curve to the initial values of both intensity and slope (Figure 5, lower center). To check this result and determine the reproducibility of the technique, a new set of measurements was made (Figure 5, lower right). This measurement consists of three independent sets of samples, each identical to those used in the initial experiment. The results clearly monitor the effect of the AAA procedure. The cluster of points in the lower left side of the plot is the indication that the AAA procedure reached its maximum effect.

An important question is whether the AAA procedure is removing only contaminating HS, as is implied by the reduction in fluorescence. It may also be removing some of the more soluble components of the degraded charcoal, assuming these are also fluorescent. Unfortunately, little is known about the diagenesis of charcoal. 


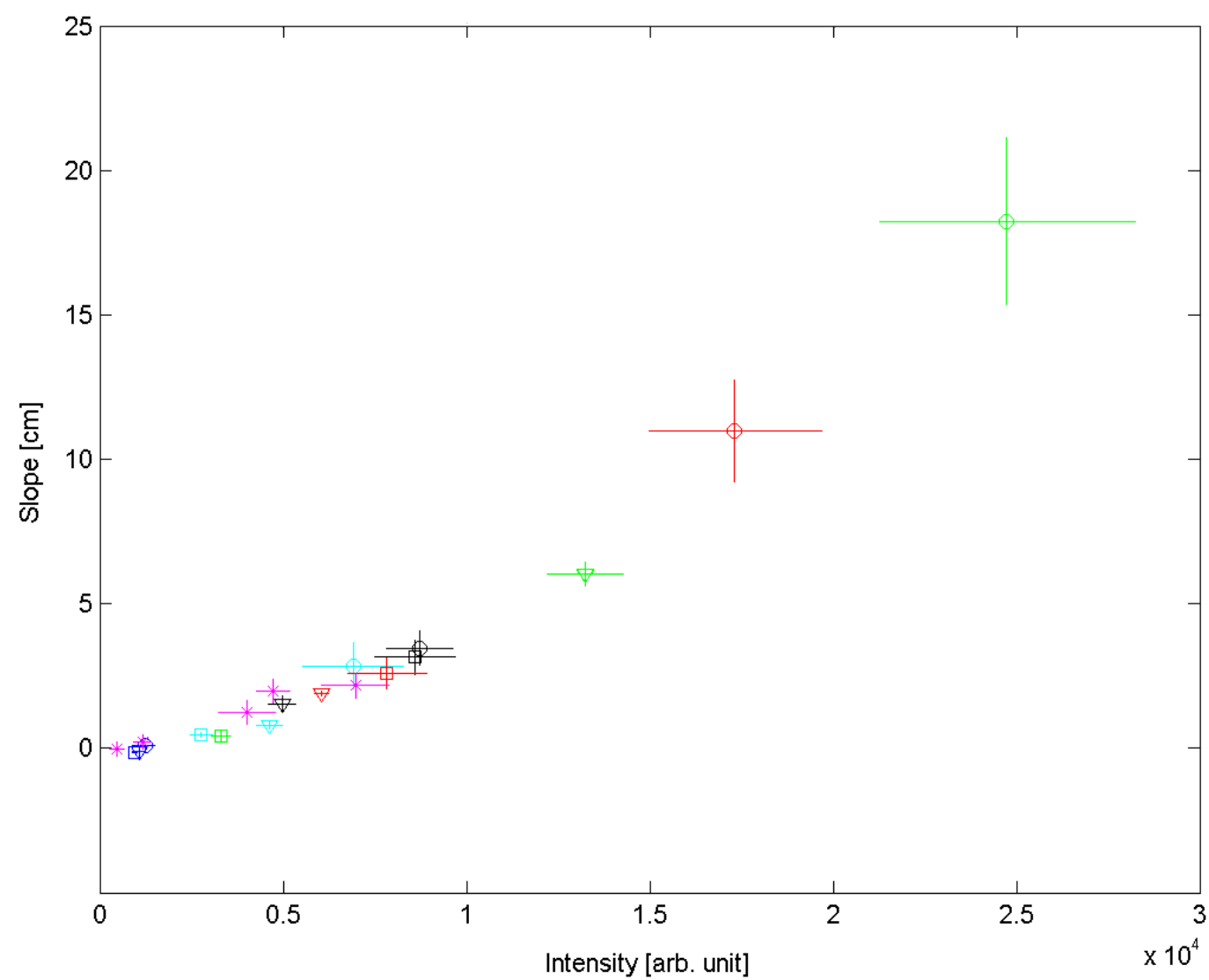

Figure 4 Plot of the slope vs. intensity values of archaeological samples. Blue = modern charcoal; green = Tel Lachish; red $=$ Tel Keissan; cyan $=$ Qumran; black $=$ Tel Hazor. Circles $=$ A fractions; triangles $=\mathrm{B}$ fractions; squares $=\mathrm{C}$ fractions. Magenta stars are the slope to intensity values of the measurements presented in Figure 3 (A) and (B).

We also note that the AAA procedure does not reduce the fluorescence and the slope to the levels found in modern charcoal. This phenomenon might be a function of the preservational state of archaeological charcoal. It may also indicate the presence of adhering sediments such as clay or of persistent HS that the AAA procedure failed to remove. These possibilities and others need to be investigated further. The observed differences between the cleaned archaeological charcoal and the modern charcoal indicate that considering charcoal cleaned by the AAA procedure as pristine, could be erroneous.

The Raman measurements can be used to demonstrate that the archaeological charcoal reached its cleanest state using the AAA procedure as seen in the samples from Tel Keissan and Tel Hazor. The measurement shows a dramatic reduction in contamination between the first and second levels of cleaning, while the third does not reduce the levels further. The second and third measurements are very similar, indicating that the AAA process reached its full effectiveness.

We recommend using this quality control method routinely before and after AAA treatment, especially for samples where accuracy is a major issue. 

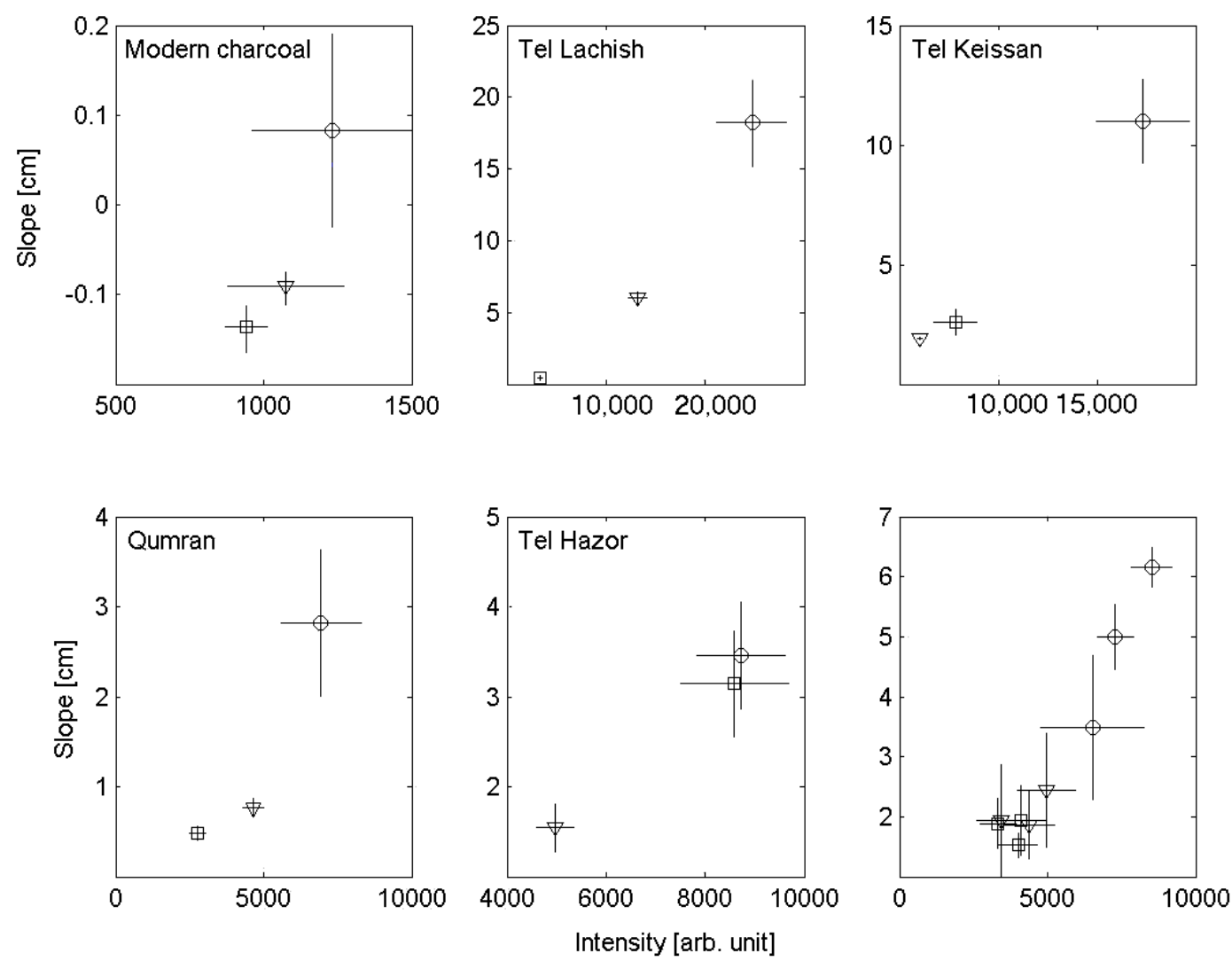

Figure 5 Plot of slope vs. intensity values of archaeological samples. Circles $=$ A fractions; triangles $=\mathrm{B}$ fractions; squares $=\mathrm{C}$ fractions. The cleaning effect of the AAA procedure can be clearly followed in most samples. Results of the reproducibility experiment are shown in the lower right plot.

\section{CONCLUSION}

To date, two assumptions are made when archaeological charcoal is dated using ${ }^{14} \mathrm{C}$. The samples are contaminated by carbon containing sediments and HS and the AAA procedure is effective in cleaning most, if not all of the contamination, leaving a pure sample. Raman shift spectrography can be used as a tool to test for and semi-quantify these two assumptions. Although this method cannot measure the exact amount of HS contamination thus providing improved accuracy and precision, the relative amount, compared with that of modern charcoal, might help determine the validity of dates measured. If an independent measurement of the $\mathrm{HS}{ }^{14} \mathrm{C}$ age is also made, the method described here might prove helpful in assessing the direction of the error.

\section{ACKNOWLEDGMENTS}

This study was supported by the Kimmel Center for Archaeological Science and a grant from the Israel Science Foundation (Grant \#302/00 to Steve Weiner and Grant \#778/00 to Dr Ilan Sharon, Hebrew University of Jerusalem). Steve Weiner holds the Dr Walter and Dr Trude Borchardt Professorial chair in Structural Biology. 


\section{REFERENCES}

Bird MI, Ayliffe LK, Fifield LK, Turney CSM, Cresswell RG, Barrows TT, David B. 1999. Radiocarbon dating of "old" charcoal using a wet oxidation, stepped-combustion procedure. Radiocarbon 41(1):127-40.

Colthup NB, Lawrence HD, Wiberley SE. 1990. Introduction to infrared and Raman spectroscopy. Third edition. Academic Press.

Darmstadt H, Pentea D, Summchen L, Roland U, Kaliaguine S, Roy C. 2000. Surface and bulk chemistry of charcoal obtained by vacuum pyrolysis of bark: influence of feedstock moisture content. Journal of Analytical and Applied Pyrolysis 53:1-17.

DeNiro MJ. 1985. Postmortem preservation and alteration of in vivo bone collagen isotope ratios in relation to palaeodietry reconstruction. Nature 317:806-9.

DeNiro MJ, Weiner S. 1988. Chemical, enzymatic and spectroscopic characterization of "collagen" and other organic fractions from prehistoric bones. Geochimica Cosmochimica Acta 52:2197-206.

Guo Y, Bustin RM. 1988. FTIR spectroscopy and reflectance of modern charcoal and fungal decayed wood: implications for studies of inertinite in coals. International Journal of Coal Geology 37:29-53.
Hatté C, Morvan J, Noury C, Martine P. 2001. Is classical acid-alkali-acid treatment responsible for contamination: an alternative proposition. Radiocarbon 43(2A): 177-82.

McGeehin J, Burr GS, Jull AJT, Reines D, Gosse J, Davis PT, Muhs D, Southon JR. 2001. Stepped-combustion ${ }^{14} \mathrm{C}$ dating of sediment: a comparison with established techniques. Radiocarbon 43(2A):255-61.

Mennella V, Monaco G, Colangeli L, Bussoletti E. 1995. Raman spectra of carbon-based materials excited at 1064 nm. Carbon 33:115-21.

Nishimaya K, Hata T, Imamura Y, Ishihara S. 1998. Analysis of structure of wood charcoal by X-ray photoelectron spectroscopy. The Japan Wood Science 44: 56-61.

Olson EA, Broecker WS. 1958. Sample contamination and reliability of radiocarbon dates. Transactions New York Academy Sciences, Series II 20:593-604.

Tuinstra F, Koenig JL. 1970. Raman spectra of graphite. The Journal of Chemical Physics 53:1126-30.

Yang Y, Wang T. 1997. Fourier transform Raman spectroscopic characterization of humic substances. Vibrational Spectroscopy 14:105-12. 